\section{Public Health} Genomics
Public Health Genomics 2013;16:127-133

DOI: $10.1159 / 000350308$
Received: October 8, 2012

Accepted after revision: February 26, 2013

Published online: April 24, 2013

\title{
Investigators' Perspectives on Translating Human Microbiome Research into Clinical Practice
}

\author{
M.J.Slashinski ${ }^{a}$ S.N. Whitney ${ }^{b} \quad$ L.S. Achenbaum ${ }^{a} \quad$ W.A. Keitel ${ }^{c}$ S.A. McCurdy ${ }^{d}$ \\ A.L. McGuire ${ }^{a}$ \\ ${ }^{a}$ Center for Medical Ethics and Health Policy, ${ }^{\text {b }}$ Department of Family and Community Medicine, and \\ 'Departments of Molecular Virology and Microbiology and Medicine, Baylor College of Medicine, and ${ }^{\mathrm{d} C e n t e r}$ for \\ Health Promotion and Prevention Research, University of Texas School of Public Health, Houston, Tex., USA
}

\section{Key Words \\ Clinical genomics · Human microbiome · Translational genomic research}

\begin{abstract}
Background: Human microbiome research has the potential to transform the practice of medicine, fundamentally shifting the ways in which we think not only about human health, illness and disease, but also about clinical practice and public health interventions. Drawing from a larger qualitative study on ethical, legal and social dimensions of human microbiome research, in this article, we document perspectives related to the translation of human microbiome research into clinical practice, focusing particularly on implications for health, illness and disease. Methods: We conducted 60 in-depth, semi-structured interviews (2009-2010) with 63 researchers and National Institutes of Health project leaders ('investigators') involved with human microbiome research. The interviews explored a range of ethical, legal and social implications of human microbiome research, including investigators' perspectives on potential strategies for translating findings to clinical practice. Using thematic content analysis, we identified and analyzed emergent themes and patterns. Results: We identified 3 themes: (1) investigators'
\end{abstract}

general perspectives on the clinical utility of human microbiome research, (2) investigators' perspectives on antibiotic use, overuse and misuse, and (3) investigators' perspectives concerning future challenges of translating data to clinical practice. Conclusion: The issues discussed by investigators concerning the clinical significance of human microbiome research, including embracing a new paradigm of health and disease, the importance of microbial communities, and clinical utility, will be of critical importance as this research moves forward.

Copyright $\odot 2013$ S. Karger AG, Basel

\section{Background}

Exploring the contributions of the more than 20 million unique microbial genes that constitute the human microbiome, the National Institutes of Health Roadmapfunded Human Microbiome Project (HMP) is shifting current paradigms of human health, illness and disease [1]. Human microbiome research is considered part of a new phase of genomic research, aptly referred to as translational genomic research, 'to capture its goal of using genomic science to develop specific interventions that can be used in clinical and public health settings to benefit

\section{KARGER}

E-Mail karger@karger.com

www.karger.com/phg
(C) 2013 S. Karger AG, Basel

$1662-4246 / 13 / 0163-0127 \$ 38.00 / 0$
Melody J. Slashinski, PhD, MPH

Center for Medical Ethics and Health Policy, Baylor College of Medicine One Baylor Plaza, Suite 310D

Houston, TX 77030 (USA)

E-Mail melody.slashinski@bcm.edu 
human health' [2, p. 7]. The ultimate goal of translating human microbiome research into clinical practice centers on producing substantive, perhaps transformative, benefits for both individual and population health [3-10]. While still in the early stages, the potential benefits of human microbiome research projects will steadily move from theory to application, where specific conditions, ranging from eczema to inflammatory bowel diseases to malnutrition, will be targeted for treatment and/or prevention. The short-term goals do not include deliverables for the practicing clinician, but those conducting this research expect that in the long-term, human microbiome research will enrich a therapeutic armamentarium $[1,2$, $8-10]$. In this article, we draw from a larger qualitative study [11] on ethical, legal and social dimensions of human microbiome research. The findings we present here document the perspectives of those involved in human microbiome research with regard to the translation of human microbiome research into clinical practice.

\section{Methods}

We conducted 60 in-depth, semi-structured interviews during the initial phase of the HMP (2009-2010) with 63 researchers and National Institutes of Health project leaders (hereafter 'investigators') engaged in human microbiome research. In-depth, semistructured interviews lasted between 30 and $90 \mathrm{~min}$, and included a series of open-ended questions exploring ethical, legal and social implications of human microbiome research. Topics discussed included investigator role; research ethics issues (e.g. informed consent, return of research results, and data sharing); potential clinical applications; ethical, legal and social implications unique to human microbiome research, and other topics as they arose (e.g. commercialization of human microbiome research [12]). To achieve a diverse sample of research, applied, and clinical expertise, we used purposive and snowball sampling strategies [13]. Purposive sampling allowed us to identify potential participants from a publicly accessible list of HMP Research Network Meeting participants, and subsequent snowball sampling allowed us to identify potential participants who were not directly involved with the HMP, but were involved with human microbiome research. These sampling strategies yielded a range of expertise, including investigators who worked on sequencing or with sequenced data, those involved with the HMP Healthy Cohort Study [14] and/or diseasespecific demonstration projects [15], and those not directly involved with the HMP, but engaged in human microbiome research.

We conducted in-person interviews at investigators' institutions, Baylor College of Medicine, or during the Human Microbiome Consortium Meetings. Interviews were digitally recorded and transcribed verbatim by a professional transcription service. We analyzed data (i.e. transcripts) using thematic content analysis [13], inductively identifying and structuring emergent themes. Two authors (M.J.S. and L.S.A.) independently coded transcripts and reached consensus in coding [16] using ATLAS.ti (v 6.2), a qualitative data analysis and management software program. ATLAS.ti files were distributed to other members of the research team for additional comment, coding and analysis.

In order to protect confidentiality of study participants, we refer to investigators using an identification number, for example (Investigator \#), following quotations or references attributable to them, and we have removed potentially identifying information such as population or disease-specific research or expertise. Investigators provided verbal consent prior to being interviewed. All procedures were reviewed and approved by the Baylor College of Medicine Institutional Review Board and the University of Texas Health Science Center Committee for the Protection of Human Subjects. A comprehensive explanation of our methods has been previously documented [11].

We asked investigators to describe what they thought would be the major clinical applications of human microbiome research, including how resulting applications might alter strategies for treatment or prevention of illness or disease, and how increasing knowledge about the human microbiome might affect clinical practice. Given the relative infancy of human microbiome research during the time we conducted these interviews (2009-2010), investigators' responses are situated in light of the knowledge available at the time. Investigators shared their perspectives regarding hopes for and expectations of the clinical implications and potential applications of human microbiome research. Three themes emerged from our interviews: (1) clinical utility of human microbiome research, (2) antibiotic use, overuse and misuse, and (3) future challenges.

\section{Results}

\section{Clinical Utility of Human Microbiome Research}

Investigators expected the clinical translation of human microbiome research would provide a valuable contribution to the theoretical and applied aspects of clinical medicine and practice, yielding 'multiple applications that will be quite relevant to science and medicine' (investigator 101), 'fundamentally shift[ing] medicine and medical treatment' (investigator 140) and revolutionizing medicine, clinical practice and how we treat human health' (investigator 162). Identifying how microbes and microbial communities impact human health, illness and disease, as well as identifying predispositions to or risks for illness or disease, were discussed as critical outcomes of human microbiome research, for example:

$[\mathrm{H}]$ opefully for us we'll be able to identify a fingerprint of [those] at higher risk for developing [disease]...It would also help us better understand the pathophysiology of the disease, which could then bring us closed to understanding, because we don't have any clue. Twenty years ago we were in exactly the same place with the same morbidity, with the same mortality with this disease as we are now, so anything we can do to bring the field forward would be an advancement. (Investigator 128) 
The potential for human microbiome research to redefine current scientific knowledge and clinical practice about the impact of microbes and microbial communities on human health, illness and disease was particularly important for those investigators working with enigmatic conditions, which investigators described as debilitating and/or fatal (e.g. chronic inflammatory bowel diseases, infectious diseases, skin disorders or maladies, or severe malnutrition). Given the current methods for diagnosing, treating or preventing these conditions were considered limited and, in some cases, severely inadequate, these investigators hoped translating human microbiome research would generate new 'objective diagnostic tool[s]' (investigator 112), and treatment approaches and prevention strategies, such as the 'development of probiotics or prebiotics for prevention and treatment' (investigator 112). Additionally, by developing a profile of an individual's microbiome, and following and documenting disease progression, investigators hoped this would one day generate rapid and more effective treatments:

One possibility [is] that there are subtle differences in the composition of the microbiome that will predispose people to a milder or severe course of the disease... We don't know, but we discover things like this from this line of research. If that turns out to be true, then as soon as someone presents with [disease] they will have their microbiome profiled, and we will know immediately what course of disease to expect, and we may be able to get people on the right kind of therapy much more quickly. (Investigator 113)

Investigators recognized human microbiome research was in an exploratory phase and would consider this phase successful if they could identify correlations between the human microbiome and health, illness and disease. Establishing cause-effect relationships was considered an ideal long-term goal, but establishing correlations in the short-term would, investigators hoped, provide instrumental information for clinicians:

...[L]ooking at a particular disease state...your hope is that you're really going to find at least some correlations, not necessarily causality, but at least correlations of the microbiome with some aspect of the disease and at least understanding that starts to open up some different areas to think about how you treat this disease state. (Investigator 118)

Strategies for preventing or treating illness or disease, and supporting maintenance of health and wellness, were discussed in terms of merging the biomedical model of identifying a physical symptom or abnormality then applying a localized treatment, with a more holistic view of human physiology and pathophysiology:

Translating Human Microbiome Research
[C] hanges in the microbiome in one part of the body can affect the microbiome in other parts of the body...one could imagine that this could lead to a somewhat more holistic view of medicine... realizing that you can't just look at one part of the body separate from all other parts. (Investigator 119)

Merging the biomedical and holistic models, investigators felt, would introduce a far more complex reality of health, illness and disease. Investigators hoped the findings from this phase of human microbiome research would encourage the adoption of an ecological perspective to frame both the positive and negative influences of microbes and microbial communities on the human body. Investigators described the human body not only as a collection of cells that carry DNA, but also as an ecosystem comprised of the human host, microbes, and microbial communities; managing the ecosystem was described as instrumental to health:

We are talking about managing an ecosystem, and many of the diseases or illnesses come from disturbances of those ecosystems. And if we find ways to manage the ecosystems in a way that maintains their service function... maybe we can maintain health or prevent disease without having to develop fancy new drugs or treatment therapies and so on. (Investigator 111)

Investigators suggested that understanding the human body as an ecosystem would fundamentally challenge current conceptualizations of health, illness and disease. An ecological perspective would provide '... a totally different way of looking at disease, because we're not talking about a single agent that causes disease. We're talking about a whole suite...co-acting together to cause what we call a disease...the fact of the matter is, we're talking about the entire human ecosystem' (investigator 153). Viewing the human body as an ecosystem introduced the importance of accounting for external environments:

Everything is connected on some level, and so as we begin to know more about our external environment, as well as the human, that does give us potentially ways in which we can begin to understand a little bit more about not only how the microbiome interacts with our body but what does that mean for our environment, and how does our environment influence us? (Investigator 118)

Investigators described a range of external environmental exposures and how these exposures shape the acquisition and operation of microbes and microbial communities, including 'food [and] stress' (investigator 125), 'socioeconomic status' (investigator 135), or neighborhood or community infrastructure(s):

The environment will change population densities, those will change infrastructures, whether it's sewage treatment or water treatment...industrial processing as far as how that influences, again, water quality, sewage treatment...it's multi-factorial and it's

Public Health Genomics 2013;16:127-133 
not to throw up your hands and say 'It's so complex we will never understand it'. But the thing is is [sic] that you definitely get a sense of: there are different concentrations of impacts or factors in different locations that can impact human health. (Investigator 162)

\section{Antibiotic Use, Overuse and Misuse}

Some investigators believed metaphors of war and sterilization, often used to describe the relationship between the human body, microbes, and microbial communities, influence the clinical use or overuse of antibiotics. These metaphors, such as 'germ warfare' (investigator 120), 'hygiene hypothesis' (investigator 145), 'death of a thousand cuts' (investigator 161), and 'sterilizing our exterior' (investigator 122) were described by some investigators as rhetorical devices used to construct bacteria as invaders, not co-inhabitants, of the human body. In order to balance the benefits of reducing pathogenic bacteria with the risks of damaging beneficial microbes and microbial communities, investigators suggested a more useful approach would be to view bacteria as 'colleagues' or 'bacterial partners' (investigator 126) that have co-evolved with humans, maintaining human health.

Investigators described developing clinical strategies to curb what they considered overuse and sometimes misuse of antibiotics as a critical outcome of this research. One strategy was to focus on the potentially beneficial properties of bacteria, harnessing those benefits as a means to restore health:

We know that when people get an infection, really it's you switch from one microbiome to another, and that if you are healthy or when you are at a steady state, if you have a certain community, then that cannot protect you against having an infection. So maybe the answer is one of instead of applying antibiotics to knock out the bad guys, maybe we should boost the good guys so that they take care of the bad guys for you. (Investigator 109)

Given the potential for antibiotic use to restore health or introduce sickness, identifying particular microbes and microbial communities, and subsequently demonstrating how these communities are altered, disturbed or eradicated by antimicrobial interventions will, some investigators argued, stimulate more cautious therapy. For example:

I can foresee a way where [illness] never has a chance to flourish. The traditional way of getting [illness] is you get treated with antibiotics. It throws your microbiome out of whack, and [illness] has sort of been there secretly hiding, but it's been kept in check by other bacteria...And then you get antibiotics that throws off the balance in your [body site], [illness] gets to grow, becomes more predominant, and then makes you sick. (Investigator 108)

Public Health Genomics 2013;16:127-133 DOI: $10.1159 / 000350308$
Some investigators with whom we spoke were exploring the clinical utility of promicrobial and paramicrobial therapies for clinical practice. These therapies were expected to promote desired microbes through the use of prebiotics and probiotics while suppressing harmful microbes, using targeted antibiotics, or simply promoting microbes known to benefit particular microbial communities. They were also exploring the clinical utility of colonizing or recolonizing bacteria for those who were either born without a particular bacteria or whose microbial community was negatively altered or destroyed, 'adding back the bacteria that they should've had to start with if there was something missing or if there was something there that shouldn't have been there' (Investigator 130).

\section{Future Challenges}

Investigators situated future challenges of translating human microbiome research into clinical practice within 3 areas: the pace of translating data from theory to application, uniqueness of an individual microbiome, and educating the general public about the benefits of some bacteria. Meaningful and clinically useful applications of scientific discoveries take time, and it was suggested the more complex the microbial community, for example the gut microbiome, the more time will be required to develop useful applications, 'I think that in body sites where it's a lot simpler, I could see clinical applications coming a lot faster...The more complex it is, I think, the longer it's going to take for any meaningful applications' (investigator 109).

Given the exploratory nature of this phase of human microbiome research, the pace of translation was described by investigators as contingent upon a number of issues that will need to be addressed prior to the development of meaningful clinical applications. The ability to reproduce research results across research sites and laboratories, for example, centered on a number of factors, including differences in protocols, methods of collecting, preparing, sequencing, and analyzing biological samples, and types of metadata collected:

...I know from our experience that different methods will just give you completely different answers. Everything from how you crack open the cells to get the DNA, to the primers you use, to what taxonomic scheme you use to bend the reads, to what you define as a read you will keep versus a read you will not use. (Investigator 151)

Investigators also discussed the potential challenges of developing clinical applications, citing the (at the time) uncertainty about commonality and variability within and across individual microbiomes, and the uncertainty 
about the unique and stable nature of each individual's microbiome:

[I]f each person is truly individual...the burden is going to be on coming up with algorithms that will be translatable to large populations, rather than a single individual. So the question is: will there be enough commonality in a patient population to come up with a generally applicable clinical intervention, or will it truly have [sic] to be totally individualized? (Investigator 142)

Investigators identified 2 issues associated with educating the general public about the clinical utility of human microbiome research. The first centered on raising awareness about the benefits of bacteria, 'I think most people...have negative ideas about bacteria, when the majority of bacteria either do not interact with us or are beneficial to us' (investigator 150), and 'I just don't think the general public is too aware of the bacteria...I just don't think people really think they actually live with a lot of bacteria everyday as part of...their life' (investigator 116). The second focused on the challenges of explaining to the general public that translating human microbiome research into clinical practice takes time, '...people have to understand that you have to walk before you can run, and you have to get some basic information before it translates to direct application...it's totally unrealistic to think that you're going to get data and suddenly have a cure of a disease process' (investigator 142).

\section{Discussion}

In this article, we presented investigators' perspectives on translating human microbiome research into clinical practice, focusing on 3 emergent themes: (1) clinical utility of human microbiome research, (2) antibiotic use, overuse and misuse, and (3) future challenges. These issues, investigators suggested, only scratch the surface but will be of critical importance as this research moves forward and as potential clinical applications are developed.

Human microbiome research is an evolving field, expected to 'address some of the most inspiring, vexing and fundamental scientific questions' [6, p. 804] through identifying 'new ways to determine health and predispositions to diseases' as well as defining 'the parameters needed to design, implement, and monitor strategies for intentionally manipulating the human microbiota, to optimize its performance in the context of an individual's physiology' (p. 804). The investigators with whom we spoke echoed these ideas, linking them to a new paradigm of health, illness and disease, and potential clinical applications to restore health and reduce disease. As the HMP concluded, and human microbiome research progresses, many of the expectations investigators shared concerning translating human microbiome research into clinical practice are being realized. For example, microbes and microbial communities have been shown to play a fundamental role in human health, illness and disease $[8,14$, 17-21]: identifying predispositions for disease risk [8], developing objective diagnostic tools [9], developing potential treatment and prevention strategies $[5,14]$, and identifying a core microbiome [21]. When human microbiome research reaches its full potential, investigators were confident the findings would lead to fundamental improvements in clinical practice and subsequently the public's health.

Investigators raised 2 important issues they believed would be critical to translating human microbiome into clinical practice. Current evidence supports their perspectives on these issues. First, investigators discussed the relationship between conceptualizations of health, illness and disease, and clinical practice. Viewing the human body as an ecosystem where bacteria are accepted as essential components of human health, or a medical ecology perspective, would effectuate tailored treatment or prevention interventions (e.g. diet, and prebiotics and probiotics) promoting beneficial microbes and resisting the intrusion of pathogens. An improved understanding of how, for example, microbial communities assemble within a human ecosystem, will undoubtedly alter future research questions, clinicians' ideas about diagnosis, treatment and prevention of illness and disease [22], and individual versus population focused interventions $[8$, 22]. Reshaping public and professional understandings of the human body and the importance of microbial communities is expected to lead to the development of tailored treatments. While investigators noted a continued role for antimicrobials, they believed these would be used with a fuller awareness of the impact on microbial flora.

Second, there is considerable controversy concerning the uniqueness of an individual's microbiome. Investigators suggested determining the uniqueness of an individual's microbiome will have profound implications for clinical practice, for example, personalized care (e.g. diet and drug intake). Previously, we documented investigators' lack of scientific consensus concerning whether or not microbial DNA was uniquely identifying, similar to human DNA [11]. This lack of consensus reflected findings reported across several publications, which suggested microbial communities in healthy individuals were diverse, highly variable and stable within and across indi- 
viduals [7, 23-26]. Fierer et al. [27] suggest microbial communities might be individually unique, and more recent published research documents the variability and stability of microbial communities $[8,14,28]$. The HMP Consortium $[8,14]$, for example, documented not only the microbial diversity within and across microbial communities (e.g. skin, gut and vagina), but also within and across each individual's microbiome.

A critical future challenge of translating human microbiome research into clinical practice will be analyzing and accurately interpreting the large amounts of data generated by the HMP and similar human microbiome research projects [29]. The development of sequencing and computational technologies $[30,31]$ will be an essential component to actualizing the clinical potential of human microbiome research, particularly for personalized or targeted therapies. These technological advancements will allow researchers to determine causal mechanisms of both health and disease, to investigate whether changes in a particular microbial community are the result or the cause of a health or disease state and to use the reference microbial genomes generated by the HMP to compare variation across different disease states [29].
Human microbiome research is expected to have a significant impact on clinical practice. While it is still unknown precisely how human microbiome research findings will be clinically relevant and explaining the actual utility of this type of research in a clinical setting at this point in time might prove difficult; investigators were confident about the long-term potential. Importantly, for diseases that are clinically heterogeneous, such as Crohn's disease, human microbiome research may soon permit prediction of severity of illness as well as response to therapy. These benefits, and others across the spectrum of human health and illness, will hopefully provide physicians with interventions that are less disruptive and more effective.

\section{Acknowledgements}

The National Institutes of Health Common Fund and the National Human Genome Research Institute (R01HG004853) supported this work. We are grateful to those who participated in this project. We would like to thank Richard Gibbs, PhD; Jim Versalovic, MD, PhD; Jill Oliver Robinson, MA; Stacey Pereira, MA, and Angela Hamaker, BA, for their research support.

\section{References}

1 Relman DA: Microbiology: learning about who we are. Nature 2012;486:194-195.

12 Juengst E, Huss J: From metagenomics to the metagenome: conceptual changes and the rhetoric of translational genomic research. Genomics, Society and Policy 2009;15:1-19.

-3 Cho MK: Translating genomics into the clinic: moving to the post-Mendelian world. Genome Med 2009;1:7.

4 Hasnain SE, Banerjee S, Ehtesham NZ: Translating advances in genomic research into clinical practice: the challenges ahead. Med Prin Pract 2010;20:392-394.

5 Rizkallah MR, Saad R, Aziz RK: The Human Microbiome Project, personalized medicine, and the birth of pharmacomicrobiomics. Curr Pharmacogenomics Pers Med 2010;8 182-193.

-6 Turnbaugh PJ, Ley RE, Hamady M, FraserLigget CM, Knight R, Gordon JI: The Human Microbiome Project. Nature 2007;449:804810.

-7 Turnbaugh PJ, Hamady M, Yatsuneko T, Cantarel BL, Duncan A, Ley RE, Sogin ML, Jones, WJ, Roe BA, Affourtit JP, Egholm M, Henrissat B, Heath AC, Knight R, Gordon JI: A core gut microbiome in obese and lean twins. Nature 2008;457:480-484.
8 The Human Microbiome Project Consortium: Structure, function and diversity of the healthy human microbiome. Nature 2012; 486:207-214.

-9 Aagaard K, Riehle K, Ma J, Segata N, Mistretta T, Coarfa C, Raza S, Rosenbaum S, Van den Veyver I, Milosavlijevic A, Gevers D, Huttenhower C, Petrosino J, Versalovic J: A metagenomic approach to characterization of the vaginal microbiome signature in pregnancy. PLoS One 2012;7:e36466.

10 Wylie KM, Mihindukulasuriya KA, Sodergren E, Weinstock GW, Storch GA: Sequence analysis of the human virome in febrile and afebrile children. PLoS One 2012;7:e27735.

-11 McGuire AL, Achenbaum LS, Whitney SN, Slashinski MJ, Versalovic J, Keitel WA, McCurdy SA: Perspectives on human microbiome research ethics. J Empir Res Hum Res Ethics 2012;7:1-14

12 Slashinski MJ, McCurdy SA, Achenbaum LS, Whitney SN, McGuire AL: 'Snake-oil,' 'quack medicine,' and 'industrially cultured organisms:' biovalue and the commercialization of human microbiome research. BMC Med Ethics 2012;13:28.

13 Creswell JW: Qualitative Inquiry and Research Design: Choosing Among Five Approaches. Thousand Oaks, Sage, 2007.
14 The Human Microbiome Project Consortium: A framework for human microbiome research. Nature 2012;486:215-221.

15 National Institutes of Health: The Human Microbiome Project: funded demonstration projects. 2013. http://www.commonfund.nih. gov/hmp/fundedresearch.aspx.

16 MacQueen K, McLellan-Lemal E, Bartholow K, Milstein B: Team-based codebook development: structure, process, and agreement; in Guest G, MacQueen K (eds): Handbook for Team-Based Qualitative Research. AltaMira, Lanham, 2008, pp 119-155.

17 Neu J, Mshvildadze M, Mai V: A roadmap for understanding and preventing necrotizing enterocolitis. Curr Gastroenterol Reports 2008;10:450-457.

18 Chen T, Yu WH, Izard J, Baranova OV, Lakshmanan A, Dewhirst FE: The human oral microbiome database: a web accessible resource for investigating oral microbe taxonomic and genomic information. Database (Oxford) 2010;2010:baq013.

19 Zhou Y, Lin P, Li Q, Han L, Zheng H, Wei Y, Cui Z, Ni Y, Guo X: Analysis of the microbiota of sputum samples from patients with lower respiratory tract infections. Acta Biochim Biophys Sin (Shanghai) 2010;42:754761 
20 Young VB, Kahn SA, Schmidt TM, Chang EB: Studying the enteric microbiome in inflammatory bowel diseases: getting through the growing pains and moving forward. Front Microbiol 2011;2:144.

21 Huse SM, Ye Y, Zhou Y, Fodor AA: A core human microbiome as viewed through $16 \mathrm{~S}$ rRNA sequence clusters. PLoS One 2012; $7: \mathrm{e} 34242$.

22 Costello EK, Stagaman K, Dethlefsen L, Bohannan BJM, Relman D: The application of ecological theory toward an understanding of the human microbiome. Science 2012;336: 1255-1262.

23 Eckburg PB, Bik EM, Bernstein CN, Purdom E, Dethlefsen L, Sargent M, Gill SR, Nelson KE, Relman DA: Diversity of the human intestinal microbial flora. Science 2005;308: 1635-1638.
24 Fierer N, Hamady M, Lauber CL, Knight R: The influence of sex, handedness, and washing on the diversity of hand surface bacteria. Proc Natl Acad Sci USA 2008;105:1799417999.

25 Nasidze I, Li J, Quinque K, Tang M, Stoneking M: Global diversity in the human salivary microbiome. Genome Res 2009;19:636-643.

26 Costello EK, Lauber CL, Hamady M, Fierer N, Gordon JI, Knight R: Bacterial community variation in human body habitats across space and time. Science 2009;326:1694-1697.

27 Fierer N, Lauber CL, Zhou N, McDonald D, Costello EK, Knight R: Forensic identification using skin bacterial communities. Proc Natl Acad Sci USA 2010;107:6477-6481.
28 Schloissnig S, Arumugam M, Sunagawa S, Mitreva M, Tap J, Zhu A, Waller A, Mende DR, Kultima JR, Martin J, Kota K, Sunyaev SR, Weinstock GM, Bork P: Genomic variation landscape of the human gut microbiome. Nature 2013;493:45-50.

29 Gevers D, Pop M, Schloss PD, Huttenhower C: Bioinformatics for the Human Microbiome Project. PLoS Comput Biol 2012; 8:e1002779.

30 National Institutes of Health Human Microbiome Project Data Analysis and Coordinating Center. 2012. http://www.hmpdacc.org/ tools_protocols/tools_protocols.php.

31 Ursell LK, Metcalf JL, Wegener Parfrey L, Knight R: Defining the human microbiome. Nutr Rev 2012;70(suppl 1):S38-44. 